\title{
BMJ Open Effects of exposure to direct and secondhand hookah and e-cigarette aerosols on ambient air quality and cardiopulmonary health in adults and children: protocol for a panel study
}

To cite: Shearston J, Lee L, Eazor J, et al. Effects of exposure to direct and secondhand hookah and e-cigarette aerosols on ambient air quality and cardiopulmonary health in adults and children: protocol for a panel study. BMJ Open 2019;9:e029490. doi:10.1136/ bmjopen-2019-029490

- Prepublication history for this paper is available online. To view these files, please visit the journal online (http://dx.doi. org/10.1136/bmjopen-2019029490).

Received 28 January 2019 Revised 4 June 2019 Accepted 5 June 2019

Check for updates

(C) Author(s) (or their employer(s)) 2019. Re-use permitted under CC BY-NC. No commercial re-use. See rights and permissions. Published by BMJ.

For numbered affiliations see end of article.

Correspondence to Jenni Shearston; js5431@cumc.columbia.edu

\section{ABSTRACT}

Introduction Use of alternative nicotine delivery systems, such as electronic cigarettes and hookahs, has increased dramatically in the USA, but limited research has been conducted on the secondhand effects of these products, especially in children. The objective of this study is to assess the cardiopulmonary effects of e-cigarette and hookah use in vaping and smoking adults, and in non-smoking/non-vaping adults and children exposed to secondhand particles and gases.

Methods and analysis This study uses a pre/post design, with four groups: two control groups (non-smoking/non-vaping and cigarette smoking) and two test groups (hookah smoking and e-cigarette vaping). Participants will be recruited by household, so that each home includes one smoking or vaping adult and one non-smoking/non-vaping adult and/or child (5-18 years). Non-smoking/non-vaping homes include an adult and child who do not smoke or vape and do not live with individuals who do. Air quality measures will be completed during a household smoking or vaping session (ambient air for non-smoking/non-vaping group), while cardiopulmonary measures and biological samples will be taken directly before and after the smoking/vaping session, and again 24 hours later, for all participants. Air quality measures include carbon monoxide, black carbon, particulate matter, trace elements, nicotine and carbonyls; cardiopulmonary measures include heart rate variability, blood pressure, pulmonary function and exhaled carbon monoxide; biological samples will assess cotinine, inflammatory cytokines and biomarkers in urine, saliva and nasal mucosa.

Ethics and dissemination This study was approved by the Institutional Review Board at New York University School of Medicine (s16-02226 and s17-01143). Special attention was given to the inclusion of children, who are likely significantly impacted by the use of these products at home, and thus should be included in research. Results of the study will be distributed at conferences, in peer-reviewed journals and to relevant public health authorities for use in developing policy.

\section{INTRODUCTION}

Tobacco use and exposure to secondhand smoke (SHS) are the leading preventable strengths and limitations of this study

- A major strength of this study design is the inclusion of children, who are likely substantially impacted by home use of hookah and e-cigarettes.

- This study includes both environmental and biological measures for individuals actively and passively exposed to smoke and aerosols from hookah and e-cigarettes, and is thus well suited to measuring actual human exposure.

- Two control groups (cigarette households and non-smoking/non-vaping households) are included in the study design, enabling results to be interpreted with nuance.

- This study does not restrict the amount of smoke or aerosol produced, how frequently the individual who uses the studied substance puffs, how deeply they inhale or the brand/type of product they use, making comparisons more challenging.

- This study will not include a random sample of individuals, and so will likely not be representative of the New York metro area.

causes of morbidity and mortality in the USA and worldwide. ${ }^{12}$ The surgeon general has stated that there is no safe level of tobacco smoke exposure, ${ }^{12}$ and an extensive body of research demonstrates the profound danger of cigarette-related SHS not only to adults, but also to children, from prenatal tobacco or childhood SHS smoke exposure. ${ }^{3-7}$ Moreover, the surgeon general has proclaimed that the most prevalent environmental tobacco smoke exposure for children occurs in the home. ${ }^{1}$ While use of conventional cigarettes has steadily declined among adults and children, ${ }^{89}$ uptake of other tobacco and nicotine delivery products, such as electronic cigarettes (e-cigarettes) and hookahs (water pipes), has increased or remained stable. ${ }^{9-11}$ Much less is known about the secondhand effects of these 
products generally, and even less is known about how any potential deleterious effects of e-cigarettes compare with those of cigarettes. Both hookahs and e-cigarettes were chosen to be included in this study as they are the leading contributors to the new epidemic of use of non-cigarette, alternative nicotine products and because they both lend themselves to near identical methodologies to investigate their influences on in-home air pollution and cardiopulmonary function.

\section{Health effects \\ E-cigarettes}

Assessing the health effects of e-cigarettes requires careful consideration of their intrinsic toxicity, their toxicity relative to that of conventional cigarettes, as well as user and device characteristics. The health effects of e-cigarettes are still inconclusive, and the long-term consequences will not be known for quite some time. However, the available literature does suggest that e-cigarette use is less harmful than conventional cigarette use, yet not entirely harmless. ${ }^{12} 13$ To date, a limited number of studies have provided information regarding potential adverse effects of e-cigarette use. The most frequently reported acute effects include mild immediate airway obstruction and inflammation, ${ }^{14}$ increased heart rate, ${ }^{15}$ throat irritation ${ }^{16}$ and asthma exacerbations. ${ }^{17}$

\section{Hookah}

Despite limited research, existing data on the health effects of hookah smoking are consistent in finding significant adverse health outcomes. A single hookah session results in a much greater amount of smoke exposure than a typical cigarette session, yielding similar levels of nicotine and much higher amounts of carbon monoxide (CO) than cigarettes. ${ }^{18}$ A meta-analysis has linked hookah use to lung cancer, oral cancer, bronchitis, cardiovascular disease and low birth weight. ${ }^{19}$ Alarmingly, users perceive hookah as a safer and less addictive alternative to cigarettes. ${ }^{20}{ }^{21}$ Evidence, however, suggests that hookah use may actually be as addictive ${ }^{18}$ and as harmful ${ }^{22} 23$ as cigarettes.

\section{Air quality and secondhand exposure E-cigarettes}

Very few studies on e-cigarette aerosols exist, aside from those in controlled exposure chambers ${ }^{14} 24$ or with smoking machines ${ }^{24-27}$ which may or may not replicate human vaping behaviour in real world settings. propylene glycol, glycerin, formaldehyde, acetaldehyde, nicotine, metals and volatile organic compounds have been found in secondhand e-cigarette aerosols ${ }^{28}$ which, unlike cigarette smoke, only consist of what the user exhales (ie, there is no aerosol produced when not using the e-cigarette). Additionally, the concentration of many of these chemicals in ambient air has been found to be 10-450 times lower than those found in cigarette smoke, depending on which toxicant is assessed. ${ }^{29-31}$ The components of e-cigarette aerosols can also vary due to differences in generation voltage applied to the heating coil and the introduction of unregulated flavourings which can vary in compounds and thus, toxicity. ${ }^{32}$

To date, there are no studies that we are aware of that report findings about the effects on air quality of e-cigarette use in homes, other than one study that found increased levels of ambient air nicotine and biomarkers of nicotine exposure in such homes and individuals residing in them. ${ }^{33}$ Only four studies have assessed secondhand exposure to e-cigarette aerosols in human subjects, ${ }^{33-36}$ measuring airborne nicotine, serum cotinine, lung function, complete blood count and inflammatory markers. However, there is a lack of knowledge on the diversity of air pollutants in homes where e-cigarettes are used, or the potential adverse health consequences to children and adults exposed to secondhand e-cigarette aerosols in such homes.

\section{Hookah}

Markedly poor indoor air quality has been demonstrated in hookah bars, where high levels of particulate matter $\left(\mathrm{PM}_{2.5}\right)$ and $\mathrm{CO}$ have been found. ${ }^{37-39}$ Furthermore, one study assessing air quality in homes while hookah was actively used found that rooms where hookah was being smoked had higher concentrations of $\mathrm{CO}, \mathrm{PM}_{2.5}$ and black carbon than rooms where cigarettes were being smoked. ${ }^{40}$ While extremely concerning, the small number of such studies contrast markedly with the extensive knowledge base on the effects of cigarette smoking on air quality. ${ }^{1}$

A similarly limited number of human studies have been conducted to examine the health effects of secondhand exposure to hookah smoke. One group found increased 3-hydroxypropylmercaptruic acid $^{41}$ and benzene ${ }^{42}$ uptake in the urine of non-smokers after they attended a hookah smoking event, suggesting that non-smoking individuals are exposed to acrolein and benzene from hookah smoke. Another found increased uptake of nicotine, tobacco specific nitrosamines, such as nicotine-derived nitrosamine ketone, and acrolein in children who lived with hookah smokers as compared with those who lived with non-smokers. ${ }^{43}$ In studies of hookah bar workers, non-smoking employees were shown to have measurable carcinogenic $^{44}$ and inflammatory biomarkers. ${ }^{45}$

\section{Study aims}

This study aims to fill many of the gaps in knowledge on the effects of e-cigarette use and hookah smoking on home ambient air quality and human health. The objectives of the study are:

1. Examine and compare the exposure atmosphere in homes where hookah water pipes or e-cigarettes are used, including the measurement of indoor levels of $\mathrm{PM}_{2.5}, \mathrm{CO}$, black carbon, nicotine, carbonyls and trace elements, in both an 'active smoking/vaping room' and in a second 'non-smoking/non-vaping' room.

2. Assess measurements of cardiopulmonary function changes and biomarkers of effect in individuals actively and passively (adults and children) exposed to 


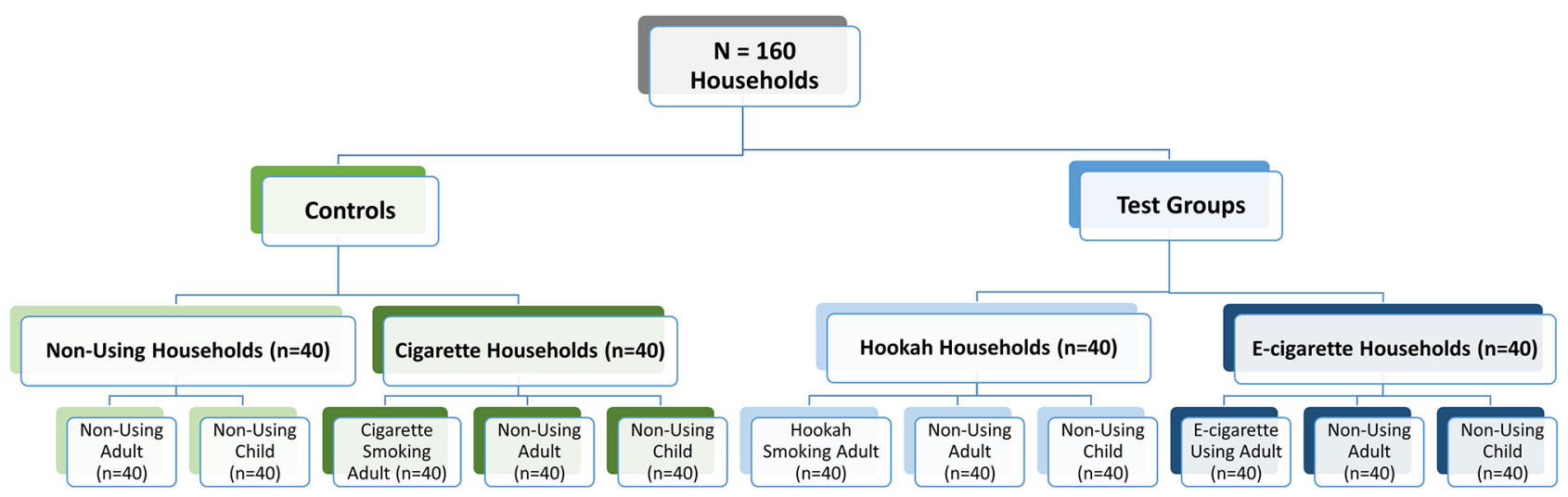

Figure 1 Study design. 'Non-using' refers to not using any tobacco product or e-cigarette product.

hookah or e-cigarette aerosols in the home, before and after a home smoking or vaping session.

We hypothesise that air quality is detrimentally altered in homes where hookahs and e-cigarettes are used, though to different degrees, and that inhalation of secondhand aerosols and gases generated by hookahs and e-cigarettes results in adverse pulmonary and cardiovascular effects in adults and children who live in such homes.

This paper will describe the protocol used to complete the above aims, in detail.

\section{METHODS AND ANALYSIS}

Data collection began in October of 2017 and is scheduled to end in autumn of 2021.

\section{Design, setting and participants}

This is a pre/post exposure study design, with two 'test' groups (hookah, e-cigarettes) and two control groups (non-smoking/non-vaping, cigarette) (see figure 1). Individuals are recruited by household, so that each household contains a minimum of two individuals, and a maximum of three who will participate in the study. A hookah, e-cigarette or cigarette household $(\mathrm{n}=40 \mathrm{each})$ will consist of a healthy, adult, male or female volunteer (21-50 years of age) who actively uses the product in their home, as well as a child (5-18 years of age) and/or adult who lives with the product user and does not use nicotine or tobacco products themselves (up to three individuals per household). Negative control households $(\mathrm{n}=40)$ will consist of a non-smoking/non-vaping adult and a non-smoking/non-vaping child who live in a household where no tobacco or nicotine products are used.

The study will consist of two home visits, approximately 24 hours apart, in which study staff come to the participants' home and collect various measures of air quality and cardiopulmonary health (figure 2). During the baseline visit, all participants in the hookah, e-cigarette and cigarette households will have health measures collected before and after an in-home hookah smoking, e-cigarette vaping or cigarette smoking session (pre-exposure and post-exposure measurements). Air quality will be assessed during the smoking/vaping session for approximately
$50 \mathrm{~min}$. For non-smoking/non-vaping controls, participants will have cardiopulmonary measures collected once at baseline, and air quality measures collected for 1 hour. For all household types, at the follow-up home visit 24 hours later, only cardiopulmonary measures will be collected.

The maximum number of participants we expect to enrol is 440 individuals across 160 households. Inclusion criteria include being a part of an exclusive hookah, e-cigarette, cigarette or non-smoking/non-vaping household, as defined in table 1, and age (adults: 21-50; children: $5-18)$. Children under the age of 5 are excluded both for ethical reasons and due to the technical difficulty of having young children complete multiple measures in a small space of time. In addition, children aged 5-9 will not be asked to complete heart rate variability (HRV) or spirometry. Exclusion criteria include regularly suffering from shortness of breath, wheeze or chest tightness; having heart disease and being pregnant. Cannabis use is not included as an exclusion criteria, but individuals are asked if they smoke or vape products other than tobacco or e-liquid, so that sensitivity analyses can be completed.

\section{Recruitment, screening and enrolment}

Several subject identification and recruitment strategies will be attempted, in order to include a range of households from different racial/ethnic groups and various neighbourhoods in the New York City and Tri-State area.

\section{Recruitment and identification}

Strategy 1: Flyers and advertisements. Advertisements will be posted in online and print media such as Facebook, Craigslist and others, and physical flyers will be posted in well-trafficked public areas, especially where individuals congregate to smoke.

Strategy 2: Snowball sampling. Study staff will reach out to individuals they know who may be interested in participating, and all study participants will be asked to forward study information to other individuals they know who may qualify or be interested in participating.

Strategy 3: Tobacco retailers. Individuals who own, work at or are a patron of local hookah bars, vape shops, shisha and tobacco retailers, or hookah/vape party host 


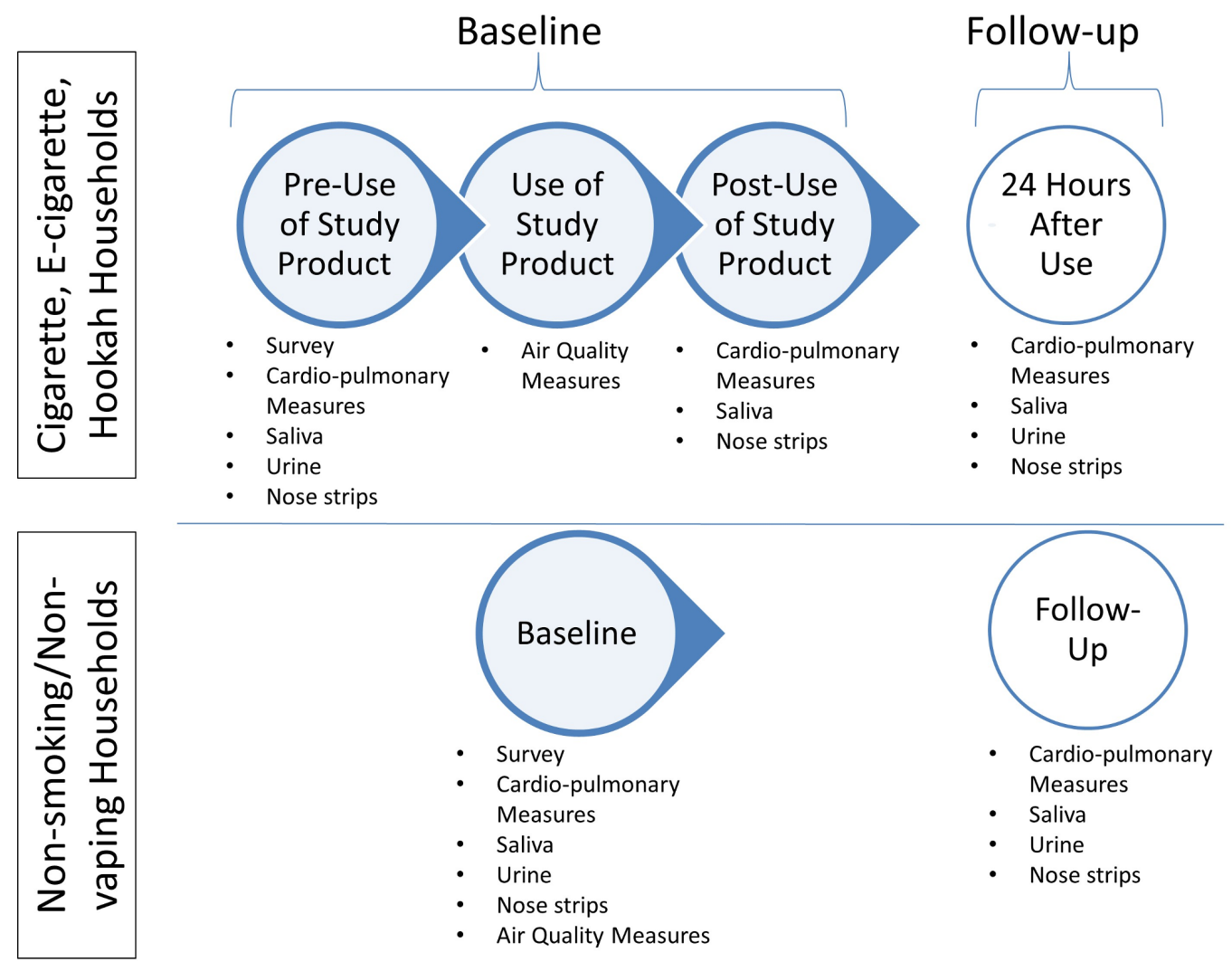

Figure 2 Measures collected at each home visit and timepoint.

organisations will be approached by study staff, who will describe the study and invite individuals to participate.

Strategy 4: Community outreach. Study staff will visit neighbourhoods in the New York City area with a high prevalence of smoking, hookah bars or vape shops and speak with local business owners, community centres and religious organisations. Study staff will explain the study and ask for permission to recruit either in person or through business cards and flyers at the location.

All participants will be compensated with a gift card, in the amount of $\$ 100$. We have chosen this amount because we feel it fairly reimburses participants for the extensive time required to participate, without being irresistibly high.

\section{Screening}

Participants who are interested in learning more about the study or determining if they are eligible must contact the research team at the study phone number or email address. A screening questionnaire has been created in the secure online platform Open RedCap V.6.10.17 (Vanderbilt University, Nashville, Tennessee, USA) which assesses inclusion and exclusion criteria and assists study staff with determining in which household type a participant may qualify. Both actively and passively exposed individuals in a potential study household are screened.

\section{Enrolment and consent}

Consent (adults) and assent (children) will be obtained at the first home visit, in English and before the onset of data collection. Study staff members will review the consent and assent forms with participants, answer any questions and confirm participant understanding of the study. Household and participant IDs will be assigned to protect participants' privacy and maintain data confidentiality.

\section{Survey}

A brief survey will be conducted with each participant independently to assess the following: demographics (age, sex, race/ethnicity), relationship of participants to each other, overall perceived health, chronic disease, frequency of hookah/e-cigarette/cigarette use, consumption of other tobacco/nicotine products, exposure to SHS and other indoor air toxins, and home parameters such as number of rooms and heat sources. Parents will be asked to help children complete the survey if children are unable to answer on their own.

\section{Air quality assessment}

Participants will be asked to refrain from conducting activities that could create indoor pollutants, such as cooking, burning a candle or spraying air freshener, for 2 hours before and during the session. In addition, participants from all households are asked to ensure that no one smokes or vapes any type of product inside the home for 48 hours before the first home visit, and between the first and second home visit, although due to the addictive nature of nicotine, it is reasonable to expect that some participants will not comply. 

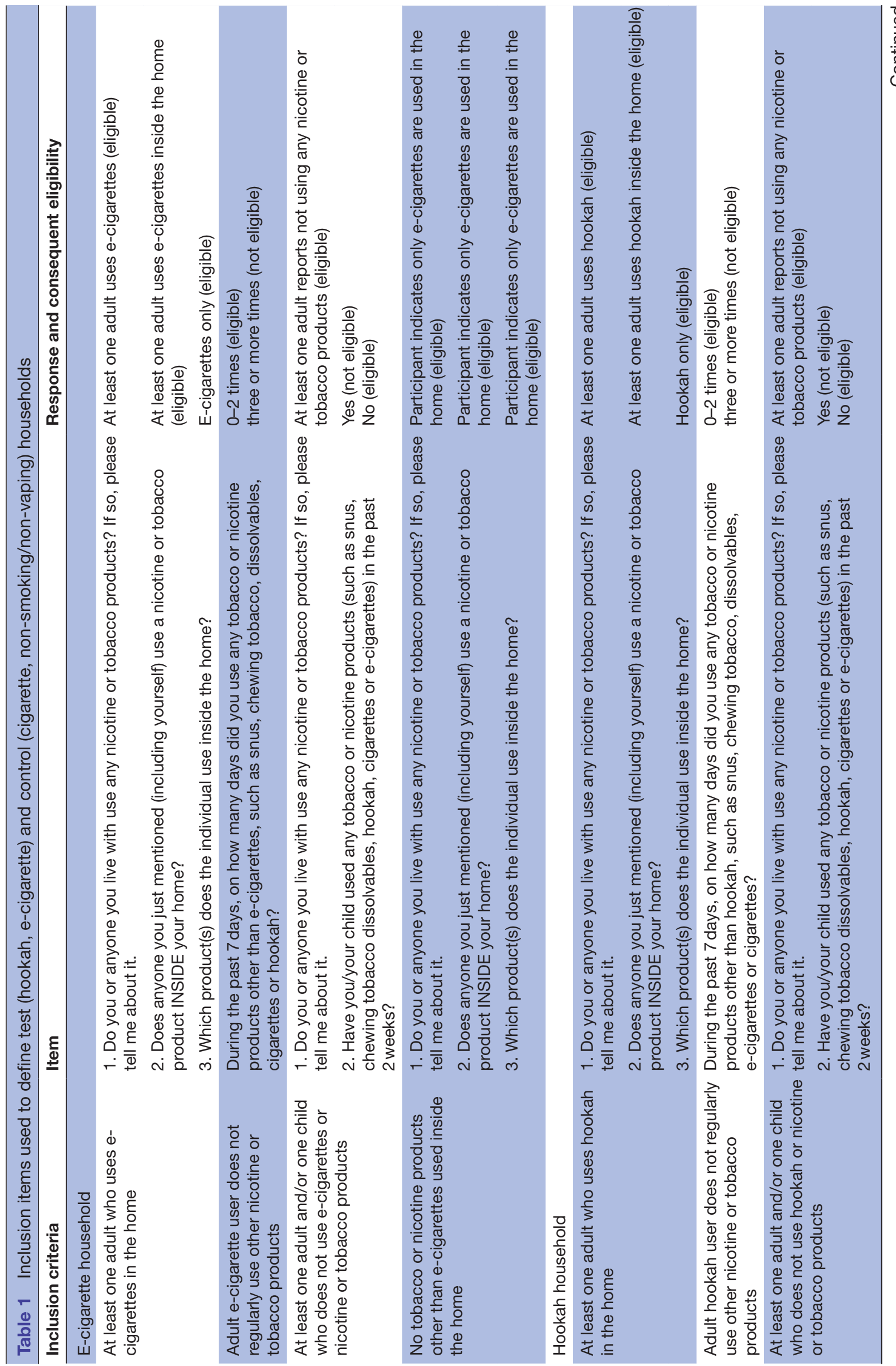

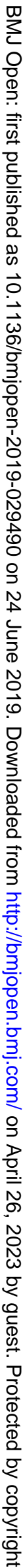




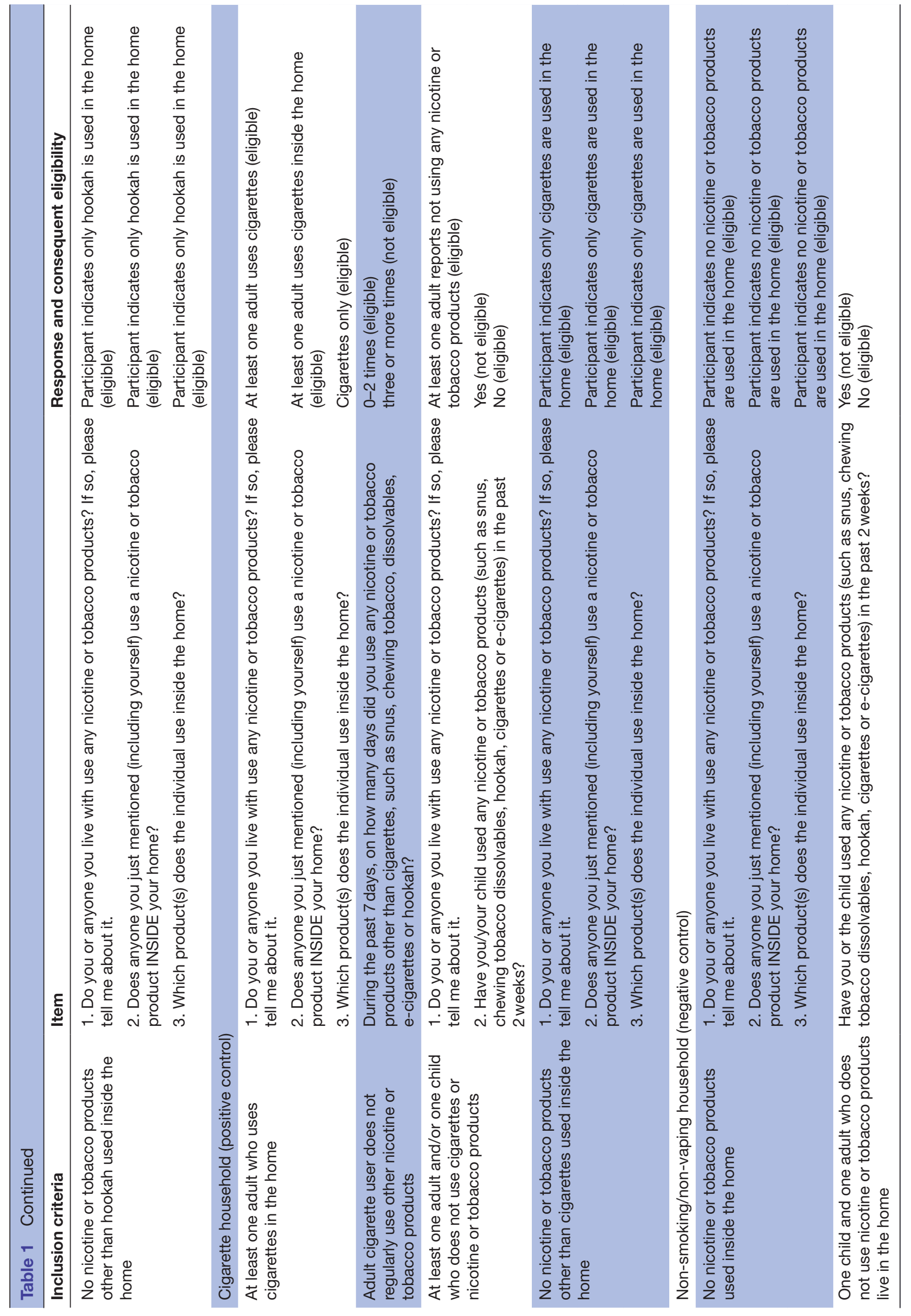

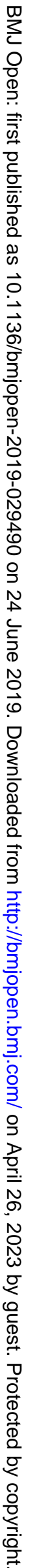


Table 2 Cardiopulmonary and air quality measures

\begin{tabular}{|c|c|}
\hline Outcome & Measurement method/equipment \\
\hline \multicolumn{2}{|l|}{ Air quality } \\
\hline Black carbon & microAeth AE51 Aetholometer (AethLabs) \\
\hline Particulate matter (2.5) & $\begin{array}{l}\text { Personal DataRAM pDR-1500 Aerosol Monitor (Thermo Fisher } \\
\text { Scientific) }\end{array}$ \\
\hline Carbon monoxide & EasyLog USB CO Data Logger (Lascar Electronics) \\
\hline Nicotine & Pump with XAD-4 sorbent tube (SKC) \\
\hline Carbonyls & Pump with silica gel sorbent tube (SKC) \\
\hline Trace elements and poly-aromatic hydrocarbons & Leland Legacy Pump with Teflon filter (SKC) \\
\hline \multicolumn{2}{|l|}{ Cardiopulmonary } \\
\hline Heart rate variability & Polar H7 chest strap; iPod; Marco Altini's HRV Logger app \\
\hline Blood pressure & Upper arm automated blood pressure monitor (Omron) \\
\hline Exhaled carbon monoxide & Micro+and piCO Smokerlyzer (Bedfont) \\
\hline Pulmonary function & $\begin{array}{l}\text { EasyOne Portable Spriometer (New Diagnostic Design); } \\
\text { TremoFlo C-100 Device oscillometer (Thorasys) }\end{array}$ \\
\hline Salivary cotinine & Saliva \\
\hline Inflammatory cytokines & Nasal mucosa samples using Leukosorb strips (Pall Scientific) \\
\hline Cotinine; 8-oxo-dG & Urine \\
\hline
\end{tabular}

Hookah, e-cigarette or cigarette households

Study staff will set up the air monitoring equipment in two rooms: the room where smoking or vaping will occur, and an adjacent room where no smoking or vaping will occur. Equipment will be placed at least one foot off the floor.

Measures for air quality in each room will consist of the following: $\mathrm{PM}_{2.5}$, measured in real time with a Data RAM pDR-1500 (ThermoScientific), and as an integrated gravimetric sample on Teflon filters (Pall) fitted with a $\mathrm{PM}_{25}$ cut PEM attached to a pump set to $10 \mathrm{~L} / \mathrm{min}$ air flow (Leland Legacy, SKC); black carbon, measured in real time with an aethalometer (AethLabs); CO, measured in real time with a continuously logging $\mathrm{CO}$ monitor (Lascar Electronics); nicotine, measured with an XAD-4 tube (\#226-094, SKC) attached to a $1 \mathrm{~L} / \mathrm{m}$ flow rate pump; and carbonyls, measured with a coated silica gel absorbent tube $(\mathrm{SKC})$ attached to a $1 \mathrm{~L} / \mathrm{m}$ flow rate pump. Trace elements will also be measured by X-ray fluorescence (XRF) analysis on the filter. A list of outcome measures and equipment can be found in table 2. Sample flow rates for all instruments will be calibrated with a DryCal instrument (SKC).

Air measures will occur for a total of approximately $80 \mathrm{~min}$ : (1) for a baseline period of approximately $15 \mathrm{~min}$ before smoking/vaping begins to allow for measurement of background air quality, (2) during smoking/ vaping and (3) for a cool-down period of approximately 15 min after smoking/vaping ends. Participants will be asked to smoke/vape as they normally do over the course of approximately $50 \mathrm{~min}$ (referred to as the smoking/ vaping session), taking as many or as few puffs as they would normally do over that time period, in order to assess impact on air quality from the 'typical' exposure in that home. No restrictions on the movement of the non-smoking/non-vaping adult and child will be made during the 50 min smoking/vaping session; children will not be required to stay in the same room as the smoking or vaping adult. The goal is to assess effects from real world exposures in the home, and many parents do not smoke or vape in the same room as their children, in order to protect them from potential harm.

Several important parameters in homes will also be recorded to control for potential factors influencing household exposure levels, including: the physical dimensions of the room in which hookahs, e-cigarettes or cigarettes are being smoked/vaped and the secondary non-smoking/nonvaping room; distance between the smoking room and non-smoking/non-vaping room; total number of rooms; type of ventilation system; type of apartment/home/building; number of open doors and windows; presence of gas stoves and heating systems; use of incense/air freshener/candles/ kitchen stove; use of air filters; past smoking/use of products in the home; number of hookah bowls/cigarettes smoked; flavour and brand of hookah, e-cigarette and cigarette products used; and nicotine content of products used.

\section{Non-smoking/non-vaping households}

Air quality measures for non-smoking/non-vaping households will be identical to those collected in smoking/ vaping households, with the following exceptions: air quality will only be measured in one room for a total of $60 \mathrm{~min}$. 


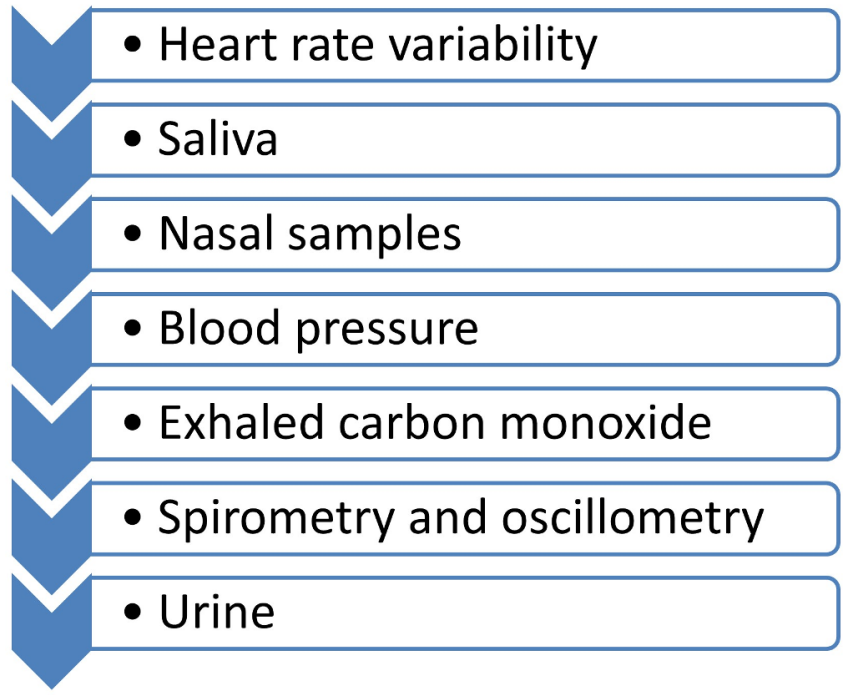

Figure 3 Preferred order of cardiopulmonary measures.

\section{Cardiopulmonary assessment}

A variety of physiological measurements and biological samples will be taken to assess acute cardiopulmonary changes in response to a smoking or vaping session, in both those who smoke or vape and in adults and children exposed only to the SHS and aerosols.

\section{Hookah, e-cigarette or cigarette households}

Cardiopulmonary measures will be assessed in all participants: immediately prior to smoking/vaping, immediately after smoking/vaping and 24 hours later.

The following measures will be collected: heart rate, HRV, blood pressure (BP), exhaled $\mathrm{CO}$ and pulmonary function. Heart rate and HRV will be continuously measured with a Polar $\mathrm{H} 7$ chest strap connected by Bluetooth to an iPhone which will log beats/min and R-R intervals using Marco Altini's HRV Logger app and an iPod or iPhone. BP will be measured with an Omron automated monitor. Three BP readings will be taken, with 1-2 min spacing between each measure to allow the brachial artery to re-dilate. Exhaled $\mathrm{CO}$ will be assessed with a Smokerlyzer. Pulmonary function will be assessed through both a portable spirometer (NDDMed) and impulse oscillometer (Thorasys) for adults, while children will be assessed through impulse oscillometry alone. Both impulse oscillometry and spirometry will be used, as spirometry can be difficult for participants, particularly children, to accurately perform, although participants will be coached by study staff to accurately perform the manoeuvres. Forced expiratory volume in $1 \mathrm{~s}$ and forced vital capacity will be calculated with American Thoracic Society (ATS) quality criteria and the mean of the three best efforts with no more than $5 \%$ variation. Age, height, ethnicity, sex and smoking status will be used to calculate the predicted pulmonary function values with appropriate databases.

The following biological samples will be collected: saliva, nasal mucosa and urine. Saliva will be collected by having participants place a swab (Sarstedt) under their tongue for $2 \mathrm{~min}$. After collection, swabs will be stored on ice during transportation to the laboratory, and then refrigerated. They will then be centrifuged to extract saliva and frozen until assayed. A sample of nasal epithelial lining fluid will be collected in all subjects, using Leukosorb medium cut into thin strips and placed inside the nostril. ${ }^{46}$ Before inserting the strip, a small amount of $5 \%$ saline solution will be sprayed into the nostril to moisten it. Strips will sit for 2 min with the nostrils clamped shut using a padded nose clip. After collection, strips will be placed in a tube and put on ice. They will be stored in a freezer until analysis for inflammatory cytokines. Participants will be given a cup to collect their own urine, and if necessary, parents will be asked to assist children who are too young to independently collect their own sample. Urine will be placed on ice during transportation, and then frozen until assayed for biomarkers of exposure (eg, polycyclic aromatic hydrocarbons (PAHs)) or effect (oxidative stress parameters). Unlike the other biological samples, urine will not be collected immediately after the smoking/vaping session because expected biomarkers are not likely to be present yet.

As collection of some measures (such as spirometry) can produce stress and may alter subsequent measurements (such as HRV), we will collect cardiopulmonary measures in the following order: HRV, saliva, nasal samples, BP, exhaled CO, spirometry, urine (see figure 3).

\section{Non-smoking/non-vaping households}

Cardiopulmonary measures for non-smoking households will be identical to that collected in smoking/vaping households, with the following exception: as there is no smoking/vaping session, measures will only be collected once from each participant during the baseline visit.

\section{Air quality sample analysis}

Gravimetric analysis for $\mathrm{PM}_{2.5}$ will be performed in NYU's environmentally controlled weighing facility using established operating procedures. Elemental quantification of trace elements by XRF (ThermoElectron) will be completed at NYU. Approximately $8 \%$ of all filters will be field and laboratory blanks. Cotinine in saliva and PAHs in urine will be measured using standard practices with the support of NYU's NIEHS Core Center. Study staff will download real-time $\mathrm{PM}_{2.5}$, black carbon and $\mathrm{CO}$ records from sampling equipment after home visits.

\section{Data analysis}

All groups will be compared on demographic characteristics, including gender, age, race, education and household income, to ensure there is not differential selection bias. This will be assessed partway through the study, so that selective recruitment of under-represented groups can be completed to re-balance study groups if needed.

The collected air quality data will be categorised into seven groups: primary smoking/vaping rooms (hookah, e-cigarette, cigarette), rooms adjacent to where smoking/ vaping occurred (hookah, e-cigarette, cigarette), and 
homes where there was no smoking or vaping. Data for all continuous variables will be summarised with descriptive statistics (eg, mean, SD, median and 95\% CI) for the $15 \mathrm{~min}$ control period before the session, the $\sim 50 \mathrm{~min}$ session, and the duration of the post-smoking/vaping session. For normally distributed variables (eg, Shapiro-Wilk test), the exposure values will be compared using an unpaired t-test or analysis of variance, as appropriate. Non-normally distributed variables will be compared using a Wilcoxon rank-sum test for medians or a Kruskal-Wallis test.

To assess cardiopulmonary changes, we will first do a bivariate analysis of changes in mean values (paired t-test) for each outcome of interest (eg, change in BP, change in HRV, etc), as well as an unpaired t-test to compare mean differences between test subjects and control subjects. We will use a mixed-effects regression model to determine associations between exposure and health outcome metrics (eg, PM and BP). The model will use pre-exposure values subtracted from post-exposure values (eg, immediate post-exposure minus pre-exposure BP), and will also include potential confounders such as gender, age, race and so on. Importantly, one of the goals of the proposed experiment is to assess the contribution of smoke/vapour components to adverse cardiopulmonary effects, yet there will likely be strong correlations among the measured pollutants. Therefore, Bayesian methods which have been successfully applied to examine multiple, correlated environmental exposures ${ }^{478}$ will also be used to study the complex mixture of SHS.

\section{Patient and public involvement}

As described above, our study will work with local community organisations during recruitment, such as local religious organisations, community centres and healthcare providers. As relationships are built, the study protocol will be modified to address any concerns brought up by these organisations or participants. Some early responses to public involvement have included asking participants if they prefer study staff of a specific gender to attend their home visit, and giving presentations to community groups as a whole before doing home visits within that community.

\section{ETHICS AND DISSEMINATION \\ Ethics}

The study design was discussed with the ethics committee extensively during institutional review. During study design and protocol development, special consideration was given to the inclusion of children and the measures that would be obtained from them. Because children are particularly impacted by exposure to SHS, and potentially to exposure from secondhand e-cigarette aerosols, we feel that it is essential to include them in the study. In order to ensure minimal risk, all data and measures collected from children fall within the range of measures they may be asked to complete in a typical doctor's visit.
Additionally, study staff are specially trained on how to interact with children, and to engage children in data collection by explaining every measure, showing the children how to use the instruments and engaging them in the process of data collection (ie, showing children how to measure the dimensions of the room as it is done, asking them to participate by pressing the 'on' button on the BP monitor). However, after careful consideration, we decided not to include children under the age of five because it would be challenging for them to complete the large range of repeat tests, some of which rely on participant effort (ie, spirometry) and would potentially result in fatigue for the children and reduced quality of data collection. Children aged 5-9 are not asked to complete spirometry or HRV, for this reason. Of note, we do not require children involved in the study to stay in the same room as adults while they are using cigarettes, hookah or e-cigarettes; instead, families are encouraged to behave as they would normally behave, including staying in the portion of the house they would normally stay in during such activities. While this does introduce some additional variability in exposure into the study, it is also more representative of true behaviours and, we believe, more ethically sound.

\section{Dissemination}

Results of the study will be disseminated in a number of ways. One to three manuscripts will be developed and submitted to public health journals for peer-review and publication. Study staff and students working on the project will be encouraged to develop and submit posters and oral presentations to appropriate domestic and international conferences. Additionally, the principal investigators of the study will also give presentations of the work to their departments and at other locations as requested. Study staff will reach out to staff at the New York Department of Health and Mental Hygiene once results are published, to encourage use in the development of policies and interventions to protect health and reduce harm.

\section{Observational versus intervention studies}

An important question for this study, as with many observational studies, is whether the process of the observation, in this case measuring air quality and taking pre/post health measures of study participants, itself introduces an indirect intervention. This may be true for our study, as participants are able to see particulate matter increase as they use their product in real-time, and may thus adjust their product use. Additionally, participants may use their product less when study staff are present in their homes, particularly if they believe the product is harmful, because they are conscientious of study staff and/or may be worried about social stigma. While this cannot be fully avoided, to counteract a potential effect, study staff are trained to encourage participants to use their product as they normally would (no more or less than usual), and to reiterate this instruction throughout the data collection visit. 


\section{Limitations}

This study has a number of limitations. First, as the design is not longitudinal and is instead a pre-post exposure design, acute changes are primarily captured. However, as participants are only eligible if they use the product inside their home, it is assumed that both smokers/vapers and those exposed to secondhand aerosols and smoke are exposed chronically, and thus biological samples collected can also be tested for biomarkers of effect, which may provide some information on long-term effects. Second, spirometry is used to assess lung function, which is an effort-based manoeuvre and is therefore subject to variability among participants, as some individuals may quickly learn how to do the manoeuvre, while others may struggle. In additional, calculating percent predicted values is dependent on participants accurately reporting height and weight. This is particularly true of children, who may have difficulty performing the manoeuvre accurately, and whose height and weight changes more rapidly. Third, there will be variation in the measured levels of exposure among participants, as different types of e-cigarettes, hookah water pipes and cigarettes will be used in different households. Additionally, among all products, we cannot control for the amount of smoke or aerosol produced, how frequently the individual who uses the substance puffs, or how deeply they inhale. Fourth, the recruitment strategies used are not representative of the population of New York City and rely on participants self-selecting by reaching out to researchers in response to advertisements. Thus, selection bias and a lack of generalisability may be present. However, demographic information from the study participants can be compared with that of smokers and vapours from the NYC Hanes surveys to determine comparability (http://www.nychanes.org). Fifth, it is possible that individuals who do not smoke or vape inside a home, but who do so outside of it, can bring toxins and remnants into non-smoking/non-vaping homes, potentially altering baseline air measures of nicotine. Finally, although participants are asked to refrain from using study products 24 hours before the home visit and between the first and second home visit, it cannot be guaranteed that participants comply. However, as exhaled $\mathrm{CO}$ will be assessed in all participants, it will be possible to determine exposure to smoke (but not to e-cigarette aerosol) before the smoking session and to remove participants from select analyses as needed. Also of note is that participants who refrain from study product use in the period before the home visit may smoke/vape more intensely during the home monitoring session, particularly if they are suffering from nicotine withdrawal, making the measured air quality and biological effects not truly representative of a typical smoking/ vaping experience.

\section{CONCLUSION}

Alternative nicotine delivery products such as e-cigarettes and hookah are rapidly emerging as serious health concerns in the USA and globally. The proposed study will contribute to evaluating the adverse effects of two of these products, with both a positive and negative control, in both direct users and those exposed to SHS and aerosols. It is likely to add much needed evidence to inform policies and future science.

\section{Author affiliations}

${ }^{1}$ Mailman School of Public Health, Environmental Health Sciences, Columbia University, New York, New York, USA

${ }^{2}$ Environmental Medicine, New York University School of Medicine, New York, USA

${ }^{3}$ Population Health, New York University School of Medicine, New York, USA

${ }^{4}$ Pediatrics, New York University School of Medicine, New York, USA

${ }^{5}$ SUNY Downstate Medical Center College of Medicine, Brooklyn, New York, USA

Contributors TG and MW conceived and designed the study. JAS, LL, SHP and MJRV contributed to study design. JAS wrote the initial manuscript draft. JE and SM revised recruitment strategies. All authors read and contributed to manuscript development and revision, and developed screening and recruitment procedures and data collection protocols.

Funding This research was supported by grants from the National Institutes of Health, grant numbers 1R21ES026996 and R01HL139239. JAS was also supported by T32 ES007322.

\section{Competing interests None declared.}

Ethics approval This study was reviewed and approved by the Institutional Review Board at New York University School of Medicine, reference numbers s16-02226 and s17-01143.

Provenance and peer review Not commissioned; externally peer reviewed.

Open access This is an open access article distributed in accordance with the Creative Commons Attribution Non Commercial (CC BY-NC 4.0) license, which permits others to distribute, remix, adapt, build upon this work non-commercially, and license their derivative works on different terms, provided the original work is properly cited, appropriate credit is given, any changes made indicated, and the use is non-commercial. See: http://creativecommons.org/licenses/by-nc/4.0/.

\section{REFERENCES}

1. Office on Smoking and Health (US): The Health Consequences of Involuntary Exposure to Tobacco Smoke: of the Surgeon General, 2006.

2. Samet JM. Tobacco smoking: the leading cause of preventable disease worldwide. Thorac Surg Clin 2013;23:103-12.

3. Daher N, Saleh R, Jaroudi E, et al. Comparison of carcinogen, carbon monoxide, and ultrafine particle emissions from narghile waterpipe and cigarette smoking: Sidestream smoke measurements and assessment of second-hand smoke emission factors. Atmos Environ 2010;44:8-14.

4. Akhtar PC, Haw SJ, Currie DB, et al. Smoking restrictions in the home and secondhand smoke exposure among primary schoolchildren before and after introduction of the Scottish smokefree legislation. Tob Control 2009;18:409-15.

5. Fong GT, Hyland A, Borland R, et al. Reductions in tobacco smoke pollution and increases in support for smoke-free public places following the implementation of comprehensive smoke-free workplace legislation in the Republic of Ireland: findings from the ITC Ireland/UK Survey. Tob Control 2006;15 Suppl 3:iii51-iii58.

6. King BA, Babb SD, Tynan MA, et al. National and state estimates of secondhand smoke infiltration among U.S. multiunit housing residents. Nicotine Tob Res 2013;15:1316-21.

7. Wilson KM, Klein JD, Blumkin AK, et al. Tobacco-smoke exposure in children who live in multiunit housing. Pediatrics 2011;127:85-92.

8. Jamal A, Homa DM, O'Connor E, et al. Current cigarette smoking among adults - United States, 2005-2014. MMWR Morb Mortal Wkly Rep 2015;64:1233-40.

9. Jamal A, Gentzke A, Hu SS, et al. Tobacco use among middle and high school Students - United States, 2011-2016. MMWR Morb Mortal Wkly Rep 2017;66:597-603.

10. Hu SS, Neff L, Agaku IT, et al. Tobacco product use among adults - united States, 2013-2014. MMWR Morb Mortal Wkly Rep 2016;65:685-91. 
11. Agaku IT, King BA, Husten CG, et al. Tobacco product use among adults-United States, 2012-2013. MMWR Morb Mortal Wkly Rep 2014;63:542-7.

12. Dautzenberg B, Garelik D. Patients with lung cancer: Are electronic cigarettes harmful or useful? Lung Cancer 2017;105:42-8.

13. Hartmann-Boyce J, McRobbie H, Bullen C, et al. Electronic cigarettes for smoking cessation. Cochrane Database Syst Rev 2016;9:Cd010216.

14. Schripp T, Markewitz D, Uhde E, et al. Does e-cigarette consumption cause passive vaping? Indoor Air 2013;23:25-31.

15. Vansickel AR, Cobb CO, Weaver MF. A clinical laboratory model for evaluating the acute effects of electronic "cigarettes": nicotine delivery profile and cardiovascular and subjective effects. Cancer epidemiology, biomarkers \& prevention: a publication of the American Association for Cancer Research. cosponsored by the American Society of Preventive Oncology 2010;19:1945-53.

16. Polosa R, Morjaria JB, Caponnetto P, et al. Effectiveness and tolerability of electronic cigarette in real-life: a 24-month prospective observational study. Intern Emerg Med 2014;9:537-46.

17. Gennimata S-A, Palamidas A, Kaltsakas G, et al. Acute effect of e-cigarette on pulmonary function in healthy subjects and smokers. European Respiratory Journal 2014;40(Suppl 56).

18. Cobb CO, Shihadeh A, Weaver MF, et al. Waterpipe tobacco smoking and cigarette smoking: a direct comparison of toxicant exposure and subjective effects. Nicotine Tob Res 2011;13:78-87.

19. Waziry R, Jawad M, Ballout RA, et al. The effects of waterpipe tobacco smoking on health outcomes: an updated systematic review and meta-analysis. Int $J$ Epidemiol 2017;46:32-43.

20. Roskin J, Aveyard P. Canadian and English students' beliefs about waterpipe smoking: a qualitative study. BMC Public Health 2009;9:10.

21. Zhou S, Van Devanter N, Fenstermaker M, et al. A Study of the Use, Knowledge, and Beliefs About Cigarettes and Alternative Tobacco Products Among Students at One U.S. Medical School. Acad Med 2015;90:1713-9.

22. Eissenberg T, Shihadeh A. Waterpipe tobacco and cigarette smoking: direct comparison of toxicant exposure. Am J Prev Med 2009;37:518-23.

23. Shihadeh A. Investigation of mainstream smoke aerosol of the argileh water pipe. Food Chem Toxicol 2003;41:143-52.

24. Geiss O, Bianchi I, Barahona F, et al. Characterisation of mainstream and passive vapours emitted by selected electronic cigarettes. Int J Hyg Environ Health 2015;218:169-80.

25. Czogala J, Goniewicz ML, Fidelus B, et al. Secondhand exposure to vapors from electronic cigarettes. Nicotine Tob Res 2014;16:655-62.

26. Lauterstein DE, Tijerina PB, Corbett K, et al. Frontal Cortex Transcriptome Analysis of Mice Exposed to Electronic Cigarettes During Early Life Stages. Int J Environ Res Public Health 2016;13:417.

27. Zhao J, Pyrgiotakis G, Demokritou P. Development and characterization of electronic-cigarette exposure generation system (Ecig-EGS) for the physico-chemical and toxicological assessment of electronic cigarette emissions. Inhal Toxicol 2016;28:658-69.

28. Cheng T. Chemical evaluation of electronic cigarettes. Tob Control 2014;23 Suppl 2(Suppl 2):ii11-ii17.
29. Tayyarah R, Long GA. Comparison of select analytes in aerosol from e-cigarettes with smoke from conventional cigarettes and with ambient air. Regul Toxicol Pharmacol 2014;70:704-10.

30. McAuley TR, Hopke PK, Zhao J, et al. Comparison of the effects of e-cigarette vapor and cigarette smoke on indoor air quality. Inhal Toxicol 2012;24:850-7.

31. Fernández $\mathrm{E}$, Ballbè $\mathrm{M}$, Sureda $\mathrm{X}$, et al. Particulate matter from electronic cigarettes and conventional cigarettes: A systematic review and observational study. Curr Environ Health Rep 2015;2:423-9.

32. Barrington-Trimis JL, Samet JM, McConnell R. Flavorings in electronic cigarettes: an unrecognized respiratory health hazard? JAMA 2014;312:2493-4.

33. Ballbè M, Martínez-Sánchez JM, Sureda X, et al. Cigarettes vs. e-cigarettes: Passive exposure at home measured by means of airborne marker and biomarkers. Environ Res 2014;135:76-80.

34. Flouris AD, Chorti MS, Poulianiti KP, et al. Acute impact of active and passive electronic cigarette smoking on serum cotinine and lung function. Inhal Toxicol 2013;25:91-101.

35. Flouris AD, Poulianiti KP, Chorti MS, et al. Acute effects of electronic and tobacco cigarette smoking on complete blood count. Food Chem Toxicol 2012;50:3600-3.

36. Tzatzarakis MN, Tsitoglou KI, Chorti MS, et al. Acute and short term impact of active and passive tobacco and electronic cigarette smoking on inflammatory markers Toxicol Lett. 2013;221 Suppl.

37. Zhou S, Weitzman M, Vilcassim R, et al. Air quality in New York City hookah bars. Tob Control 2015;24:198:e193-e198.

38. Fiala SC, Morris DS, Pawlak RL. Measuring indoor air quality of hookah lounges. Am J Public Health 2012;102:2043-5.

39. Cobb CO, Vansickel AR, Blank MD, et al. Indoor air quality in Virginia waterpipe cafes. Tob Control 2013;22:338-43.

40. Weitzman M, Yusufali AH, Bali F, et al. Effects of hookah smoking on indoor air quality in homes. Tob Control 2016;26.

41. Kassem NOF, Kassem NO, Liles S, et al. Acrolein exposure in hookah smokers and non-smokers exposed to hookah tobacco secondhand smoke: implications for regulating hookah tobacco products. Nicotine Tob Res 2018;20:492-501.

42. Kassem NO, Kassem NO, Jackson SR, et al. Benzene uptake in Hookah smokers and non-smokers attending Hookah social events: regulatory implications. Cancer Epidemiol Biomarkers Prev 2014;23:2793-809.

43. Kassem NO, Daffa RM, Liles S, et al. Children's exposure to secondhand and thirdhand smoke carcinogens and toxicants in homes of hookah smokers. Nicotine Tob Res 2014;16:961-75.

44. Moon KA, Rule AM, Magid HS, et al. Biomarkers of secondhand smoke exposure in waterpipe tobacco venue employees in istanbul, moscow, and cairo. Nicotine Tob Res 2018;20:482-91.

45. Zhou S, Behrooz L, Weitzman M, et al. Secondhand hookah smoke: an occupational hazard for hookah bar employees. Tobacco Control 2015. In press.

46. Rebuli ME, Speen AM, Clapp PW, et al. Novel applications for a noninvasive sampling method of the nasal mucosa. Am J Physiol Lung Cell Mol Physiol 2017;312:L288-L296.

47. Greenland S. Principles of multilevel modelling. Int J Epidemiol 2000;29:158-67.

48. MacLehose RF, Dunson DB, Herring AH, et al. Bayesian methods for highly correlated exposure data. Epidemiology 2007;18:199-207. 